\title{
Simple Removal of Ventriculoatrial Shunt and Simultaneous Ventriculoperitoneal Shunt Revision: Short Communication
}

\author{
Ryo Oike ${ }^{1, \odot ~}$ Yasuaki Inoue $^{2}$ Kazuhito Matsuzawa ${ }^{1}$ \\ ${ }^{1}$ Department of Neurosurgery, Nadogaya Hospital, Chiba, Japan \\ 2Department of Neurosurgery, Brigham and Women's Hospital, \\ Harvard Medical School, Boston, Massachusetts, United States \\ Indian J Neurosurg 2021;10:152-154.
}

Address for correspondence Ryo Oike, MD, Department of Neurosurgery, Nadogaya Hospital, 2-1-1 Shin-Kashiwa, Kashiwa, Chiba 277-0084, Japan (e-mail: ryo.st2b@gmail.com).

\begin{abstract}
Background Ventriculoatrial shunt (VAS) is a common alternative treatment option for hydrocephalus in patients with ventriculoperitoneal shunt (VPS) failure. Most previous reports on VAS discuss the atrial-related complications and none focus on simple removal (i.e., without specialized equipment). We report a case of simple VAS removal and simultaneous VPS revision, with no obvious shunt-related cardiac complications.

Case presentation The patient was an 87-year-old female who had received a VAS for idiopathic normal pressure hydrocephalus 6 years prior. She developed a right thalamic hemorrhage with intraventricular hemorrhage and was admitted to our hospital. She had a recurrence of the hydrocephalus and was diagnosed with shunt malfunction, due to simple obstruction without obvious shunt-related cardiac complications. The VAS was simply and safely removed, and a VPS was simultaneously placed, as per the usual procedure in our institution. She remains well with no evidence of complications on postoperative day 10 .

Discussion Since VAS is mostly used in pediatric cases that are difficult to treat with VPS, the duration of time elapsed allows VAS catheters to form strong adhesions with

Keywords

- Ventriculoatrial shunt

- ventriculoperitoneal shunt

- hydrocephalus

the surrounding cardiac tissue. Therefore, the simple removal of VAS is usually not straightforward.

Conclusion If the follow-up period is short and there are no specific cardiac complications at the time of replacement, VAS can be safely removed and VPS can be spontaneously placed, without any specialized surgical techniques or equipment.
\end{abstract}

\section{Introduction}

The ventriculoatrial shunt (VAS) was first introduced by Nulsen and Spitz in 1952. With the contribution of Pudentz in the evolution of the shunt valve, the VAS became popular for the treatment of hydrocephalus until the 1970s. However, long-term severe cardiac and renal complications have been reported, such as complications associated with tricuspid

published online July 15, 2021
DOI https://doi.org/

$10.1055 / \mathrm{s}-0041-1730131$ ISSN 2277-954X lesions, endocarditis, thrombosis, the catheter traversing the foramen ovale, and pulmonary hypertension. ${ }^{1,2}$ In previous reports, shunt removal by sternotomy was recommended for the treatment of these complications, while an endovascular approach is common for the removal of a broken VAS catheter. ${ }^{1,3,4}$ Thus, the VAS was established as an alternative treatment when the other options were not feasible. In recent years, however, some have reported that the VAS

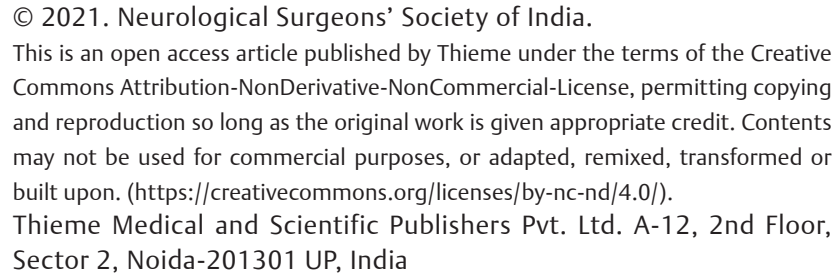
and reproduction so long as the original work is given appropriate credit. Contents may not be used for commercial purposes, or adapted, remixed, transformed or built upon. (https://creativecommons.org/licenses/by-nc-nd/4.0/). Thieme Medical and Scientific Publishers Pvt. Ltd. A-12, 2nd Floor, Sector 2, Noida-201301 UP, India 
should be considered the treatment of choice for hydrocephalus in older patients, because in their studies, patients with VAS were less likely to experience shunt obstruction than those with VPS. ${ }^{5}$ Accordingly, we are more likely to encounter older patients with VAS. There are no previous reports of the simple removal of VAS without any specialized techniques or equipment with simultaneous shunt revision using VPS. In the present case, we report a patient who underwent simple removal of the VAS and simultaneous VPS revision without any shunt-related cardiac complications.

\section{Case Presentation}

An 87-year-old, otherwise healthy female, had undergone implantation of a VPS for the treatment of idiopathic normal pressure hydrocephalus 6 years prior. She developed mild chronic subdural hematoma through overdrainage, but neither revision nor evacuation of hematoma was performed. She presented with left hemiparesis and was transferred to our hospital, and right thalamic hemorrhage with intraventricular hemorrhage was diagnosed. One month after admission, head CT scan revealed the hematoma had resolved with ventriculomegaly ( - Figs. 1 A, B). Transthoracic echocardiography (TTE) demonstrated no obvious cardiac complications. The reservoir of the VAS would not pump up after it was pushed in, suggesting malfunction of the shunt system, especially on the cranial side. She underwent VAS removal by patching of the internal jugular vein with fascia, and a VPS was performed simultaneously as per the usual procedure in our institution ( - Fig. 2). She remains well with no evidence of complications on postoperative day 10 . The patient has provided informed consent for publication of the case.

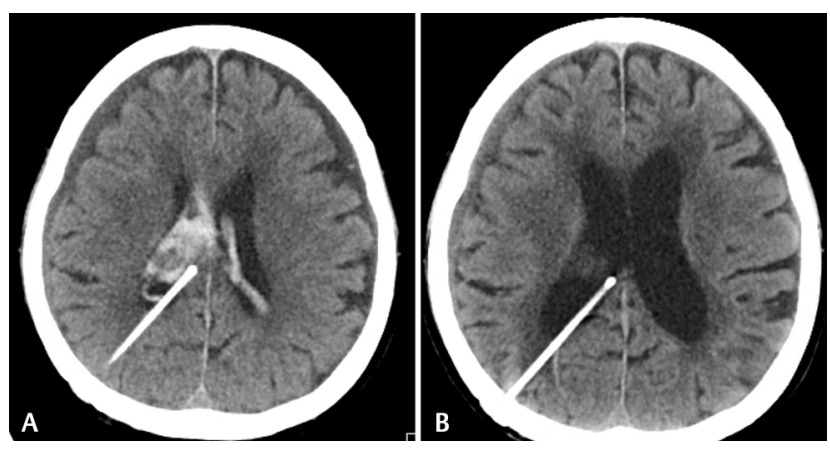

Fig. 1 Preoperative axial CT scan (A) on admission; (B) 1 month after admission, indicating a recurrence of hydrocephalus.

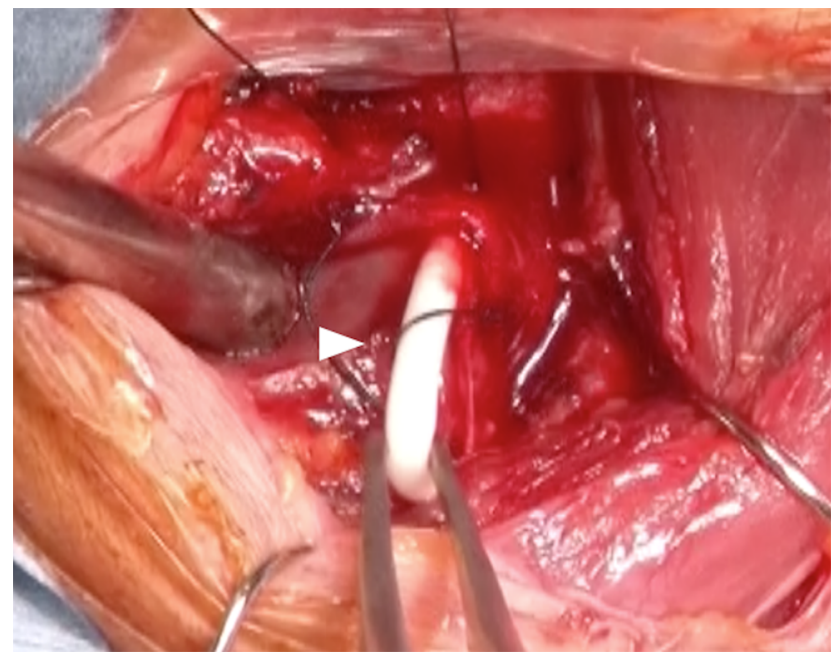

Fig. 2 Intraoperative photographs of the internal jugular vein at the insertion site of the shunt catheter (white arrowhead).

\section{Discussion}

The removal of a long-term implanted VAS is not recommended, because of the possibility of severe adhesion to the right atrium or formation of an intracardiac thrombus. ${ }^{6,7}$ Our review of the literature identified only three cases of simultaneous VAS removal and VPS revision in adulthood (-Table 1) $)^{2,4,7}$ The articles were identified via a PubMed search using the keywords "ventriculoatrial shunt," "complications," and "shunt revision," alone and in combination. In two of the three cases, the VAS removal was achieved through sternotomy, and the revision was performed several days later. ${ }^{2,7}$ The third case underwent endovascular treatment, wherein the distal catheter was removed intravenously, and then the remaining shunt system was connected to a new peritoneal catheter; this does not constitute simple removal. ${ }^{4}$ Natarajan et al recommend follow-up using TTE for patients who had undergone VAS implantation more than 10 years ago. ${ }^{8}$ Paradini-Santos et al reviewed reports of VAS complications and suggested that, if the catheter has only recently been inserted, simple traction on the distal catheter may be sufficient for removal. ${ }^{1}$ These reports suggest that if there are no obvious VAS-related complications identified on TTE, safe removal of the VAS is possible.

According to Alvi et al, VAS accounted for $0.95 \%(n=130)$ of all shunting procedures in patients over 60 years of age

Table 1 Summary of previous case reports on surgery for complication of VAS

\begin{tabular}{|l|l|l|l|l|l|}
\hline Author (year) & Age & Sex & Complication & Treatment & Revision \\
\hline Cowan and Allen $^{2}$ & 52 & $\mathrm{M}$ & Retrograde cardiac migration & Ligation of IJV & VAS on the contralateral side \\
\hline Tonn et al $^{4}$ & 26 & $\mathrm{~F}$ & Pulmonary embolism & Endovascular & VPS \\
\hline Gopal and Peethambaran $^{7}$ & 41 & $\mathrm{M}$ & Endocarditis & Sternotomy & VAS \\
\hline
\end{tabular}

Abbreviations: F, female; M, male; IJV, internal jugular vein; VAS, ventriculoatrial shunt; VPS, ventriculoperitoneal shunt. 
from 2007 to $2017 .{ }^{9}$ In recent years, however, there have been more reports that there is no difference in long-term complications between VPS and VAS in older patients. Rymarczuk et al reported that the most common etiology of shunt malfunction is proximal catheter failure, ${ }^{10}$ and Paradini-Santos et al reported that the most common complications of shunt malfunction were shunt obstruction or disconnection. ${ }^{1}$ In other words, the number of cases in which removal and replacement are necessary without VAS-related complications is expected to increase. The present study, based on these reports, demonstrated the safe removal of VAS with only basic hemostasis via pressure on the fascia of the internal jugular vein at the distal catheter insertion site and the usual revision of the VPS.

\section{Conclusion}

We reported that VAS removal in patients without VAS-related cardiac complications could be performed without specialized techniques and that simultaneous VPS was possible.

\section{Funding}

None.

\section{Conflict of Interest}

None declared.

\section{Acknowledgement}

The authors thank all the members of our team for their generous support during the course of the study.

\section{References}

1 Pradini-Santos L, Craven CL, Watkins LD, Toma AK. Ventriculoatrial shunt catheter tip migration causing tricuspid regurgitation: case report and review of the literature. World Neurosurg 2020;136:83-89

2 Cowan MA, Allen MBJr. Retrograde migration of the venous catheter as a complication of ventriculoatrial shunts in adults. Case report. J Neurosurg 1971;35(3):348-350

3 Curry JL. Recovery of detached intravascular catheter or guide wire fragments. A proposed method. Am J Roentgenol Radium Ther Nucl Med 1969;105(4):894-896

4 Tonn P, Gilsbach JM, Kreitschmann-Andermahr I, Franke A, Blindt R. A rare but life-threatening complication of ventriculo-atrial shunt. Acta Neurochir (Wien) 2005;147(12):1303-1304

5 Hung AL, Vivas-Buitrago T, Adam A, et al. Ventriculoatrial versus ventriculoperitoneal shunt complications in idiopathic normal pressure hydrocephalus. Clin Neurol Neurosurg 2017;157:1-6

6 Bastian D, Fessele K, Bednarski P, Göhl K. Interventional laser-assisted extraction of an infected trapped ventriculoatrial shunt based on techniques for pacemaker lead explantation. J Neurol Surg A Cent Eur Neurosurg 2016;77(1):73-76

7 Gopal VV, Peethambaran AK. Rare sequelae following ventriculoatrial shunt: Case report and review of literature. Asian J Neurosurg 2016;11(2):173

8 Natarajan A, Mazhar S. Right heart complications of ventriculoatrial shunt. Eur Heart J 2011;32(17):2134

9 Alvi MA, Brown D, Yolcu YU, et al. Predictors of adverse outcomes and cost after surgical management for idiopathic normal pressure hydrocephalus: Analyses from a national database. Clin Neurol Neurosurg 2020;197:106178

10 Rymarczuk GN, Keating RF, Coughlin DJ, et al. A comparison of ventriculoperitoneal and ventriculoatrial shunts in a population of 544 consecutive pediatric patients. Neurosurgery 2020;87(1):80-85 